\title{
Expression of achaete and scute genes in Drosophila imaginal discs and their function in sensory organ development
}

\author{
Susana Romani, ${ }^{1,3}$ Sonsoles Campuzano, ${ }^{1,3}$ Eduardo R. Macagno, ${ }^{2}$ and Juan Modolell ${ }^{1}$ \\ ${ }^{1}$ Centro de Biología Molecular, Consejo Superior de Investigaciones Científicas and Universidad Autónoma de Madrid, 28049 \\ Madrid, Spain; ${ }^{2}$ Department of Biological Sciences, Columbia University, New York, New York 10027 USA
}

\begin{abstract}
Several kinds of sensory organs (SOs) appear in stereotyped positions on the adult Drosophila cuticle. The generation of these SOs requires the activity of the achaete $(a c)$ and scute (sc) genes. To investigate whether ac and $s c$ also provide spatial information for the positioning of SOs, we have analyzed the patterns of expression of these genes in the wing imaginal disc around the time that SO precursors are being specified. We find that expression coincides with and is restricted to areas of the disc where these precursors are known to be located. In the loss-of-function $s c^{6}$ mutant, $s c$ RNA is depleted in a single area located in the region where the precursor for the supressed macrochaeta should be found. Moreover, some, and probably all, SOs require expression of these genes to reach the earliest detectable differentiated state. These and other results presented here, together with the finding that expansion of the areas of $a c$ and/or $s c$ expression causes the development of ectopic SOs, indicate that $a c$ and $s c$ promote the determination of $S O$ precursors and delimit the regions of the imaginal discs where they can develop.
\end{abstract}

[Key Words: Sensory organs; achaete; scute; Drosophila]

Received February 17, 1989; revised version accepted April 18, 1989.

The wings, heminota, and thoracic pleura of Drosophila derive from two pouches of ectodermal cells known as the imaginal wing discs (WDs). Each WD anlage consists of a nest of $\sim 50$ cells, set apart during embryogenesis, which, in the larva and 1-day-old pupa, divide every 8.5 hr until the disc contains $\sim 50,000$ cells (García-Bellido and Merriam 1971a). In pupae, WDs evaginate and undergo morphological modifications in order to give rise to the adult structures. Most WD cells eventually form the epidermis, which will secrete the cuticle. However, $\sim 500$ cells enter a neural developmental pathway to form sensory organs (SOs) which are bristles (macro- and microchaetae) or sensilla of other types (campaniformia, trichoidea, etc.). As it occurs in other insects, it is thought that in Drosophila each SO originates from a precursor cell that undergoes two differential divisions (for review, see Bate 1978). One pair of sister cells gives rise to the external part of the SO (basal cell and chaeta or another type of sensillum). The other pair of cells differentiates into a neuron and a glial cell. The type and position of these SOs form a stereotyped pattern that provides an excellent model to study pattern formation.

The achaete-scute complex (AS-C), which plays an important role in the generation of this pattern (for review, see Ghysen and Dambly-Chaudière 1988), spans $90 \mathrm{~kb}$ of DNA and contains several transcription units (Campuzano et al. 1985; Ruiz-Gómez and Modolell

${ }^{3}$ The order of these authors is arbitrary.
1987), only four of which seem important for the neurogenic functions of the complex (Alonso and Cabrera 1988; F. González and J. Modolell, unpubl.). These units have been named T5, T4, T3, and T1a $(=\mathrm{T} 8)(\mathrm{Campu}-$ zano et al. 1985; Alonso and Cabrera 1988; Balcells et al. 1988). However, because a considerable body of evidence indicates that they correspond to the genetically defined achaete (ac) (T5), scute (sc) (T4), lethal of sc (1'sc) (T3) and asense (ase) (Tla) functions of the complex (for review, see Ghysen and Dambly-Chaudière 1988), they will hereafter be referred to by the abbreviated name of the corresponding function. They encode structurally related proteins with a domain similar to regions of the mammalian myc (Villares and Cabrera 1987; Alonso and Cabrera 1988; F. González and J. Modolell, unpubl.), MyoD1 (Davis et al. 1987), and immunoglobulin enhancer binding proteins (Murre et al. 1989) and the product of the Drosophila daughterless (da) gene (Caudy et al. 1988b). Like the AS-C, $d a$ is necessary for SO development (Caudy et al. 1988a). This similarity suggests that all of these genes may be members of a new family of transcriptional regulators (Alonso and Cabrera 1988; Caudy et al. 1988b; Lech et al. 1988; Murre et al. 1989).

The $a c$ and $s c$ genes are most important for the development of the adult pattern of SOs (García-Bellido and Santamaría 1978). Their hypomorphic condition causes the loss of SOs from allele-specific positions (García-Bellido 1979). On the WD derivatives, $a c$ and $s c$ genes promote development of different sets of SOs, although 
some organs, like a fraction of the notum microchaetae, can develop in the presence of either gene alone /GarcíaBellido 1979; Ruiz-Gómez and Modolell 1987; J. Modolell, unpubl.). The lesions associated with $a c$ and $s c \mathrm{mu}$ tations may map far from the corresponding structural genes, up to $50 \mathrm{~kb}$ for some $s c$ mutations (Campuzano et al. 1985). This suggests that the control of $a c$ and $s c$ expression probably involves large regions of DNA, which may harbor enhancer-like elements that respond to spatial cues and stimulate $s c$ transcription in specific regions of the imaginal discs (Ruiz-Gómez and Modolell 1987). Accordingly, the specificity of the $s c$ phenotypes would depend on the particular interference of each mutation with the $s c$ promoter region and/or the particular set of enhancers inactivated (Ghysen and Dambly-Chaudière 1988). On the other hand, it has been shown that ectopic expression of $a c$ and $s c$ induces development of extra SOs in ectopic positions (Campuzano et al. 1986; Balcells et al. 1988). These data suggest that the spatial distribution of $a c$ and $s c$ products plays a key role in establishing the pattern of SOs.

To investigate this role, we have resorted to the Drosophila WD, one of the few anlage whose detailed fate map is known (Bryant 1975). We have determined the sites of $a c$ and $s c$ expression in this disc and have compared them with the location of SO precursor cells. We have found a coincidence between both sets of sites. Also, we have investigated the modifications of the expression patterns and their correlations with the appearance of SO precursors in mutant flies. The results support a role of $a c$ and $s c$ in the determination and early differentiation of SOs and in the specification of positions where SOs develop.

\section{Results}

\section{Expression of AS-C genes in late third-instar larvae}

AS-C genes are expressed in the embryo and during most of the third-instar larval and early pupal stages (Campuzano et al. 1985; Alonso and Cabrera 1988; S. Romani and S. Campuzano, unpubl.). The second period of expression coincides with the developmental stages when adult SOs are determined (García-Bellido and Merriam 1971a; García-Bellido and Santamaría 1978). Recently, we found that in late third-instar (LTI) larvae, ase and l'sc are expressed almost exclusively in the central nervous system (CNS). In the imaginal discs, their mRNAs are restricted to a small number of scattered cells or to an occasional cluster of cells (not shown). This scarcity of expression is consistent with the absence $\left(I^{\prime} s c\right)$, or very small (ase) effect, of the deletions of these genes on the adult pattern of SOs /García-Bellido and Santamaría 1978; Campuzano et al. 1985; L. Leyns and A. Ghysen, pers. comm.).

In contrast, the $s c$ gene is expressed in many sites of the imaginal discs and, to a smaller extent, in the CNS (Fig. 1). The ac gene is expressed also, albeit less abundantly, in all discs, but its RNA was not detected in the CNS (not shown). The distribution of $a c$ and $s c$ RNA in the WD was studied in detail because a fate map of this

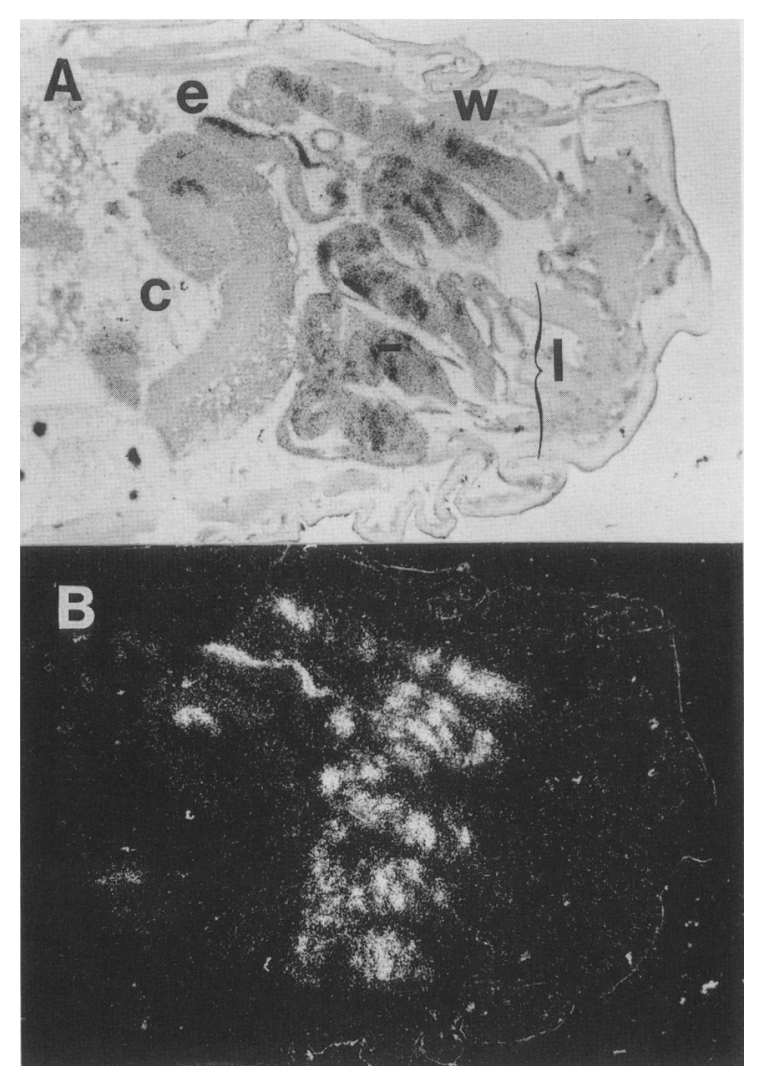

Figure 1. Expression of $s c$ in LTI larvae. Bright $(A)$ and dark $(B)$ fields of a parasagittal oblique section. $s c$ expression occurs exclusively in imaginal discs and CNS. Note the many sites of expression in the discs. (w) Wing disc; (l) leg discs; (e) eye-antenna disc; (c) central nervous system.

disc is available (Bryant 1975; Fig. 3B) and the pattern of SOs it gives rise to, in wild-type and mutant flies, is the best characterized.

$s c$ and $a c$ are expressed in may sites of the WD (Fig. 2A,B,E,F). Hybridization occurs in separate clusters of cells. A few regions of the disc also show a weaker, more diffuse label (Fig. 2A,B,F). Because of the complexity of the patterns of expression and the convoluted structure of the disc, it is difficult to show all hybridization sites in only a few sections. Consequently, to represent the complete patterns, we resorted to a computer-aided three-dimensional reconstruction of labeled WDs starting from a complete series of hybridized sections (Fig. 3). The models and the sections have shown that the $a c$ and $s c$ patterns are very similar, although the $s c$ RNA is more abundant and seems to extend, in some places, over more cells.

The regions of expression (Figs. 2A,B,E,F and $3 \mathrm{~A}, \mathrm{C}, \mathrm{D}$ ) coincide remarkably with the locations of SO precursors, according to the fate map (Fig. 3B). Thus, prominently labeled sites correspond to the presumptive anterior, but not the posterior, wing margin, which carries a large number of innervated bristles in the adult; the tegula region, which contains $>20$ sensilla campaniformia and trichoidea and several chaetae, and the middle of the second transverse fold, which corresponds to the dorsal 


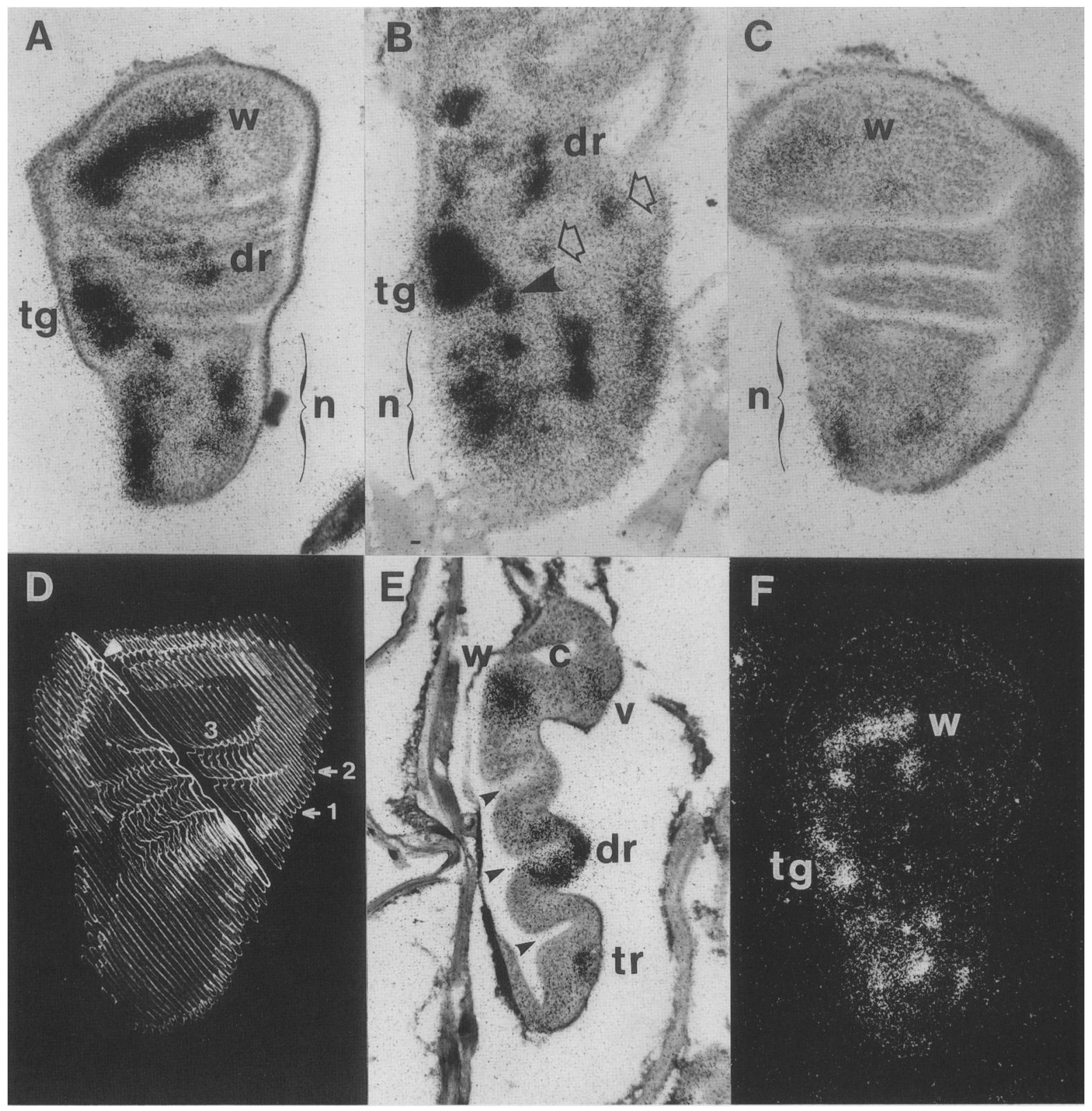

Figure 2. Localization of $s c(A, B, E)$ and $a c(F)$ RNA in wild-type WD sections, of $s c$ RNA in an $\operatorname{In}(1) s c^{4}$ WD section $(C)$, and a computer-generated reconstruction of a WD from unlabeled serial sections $(D)$. Sections in $A$ and $C$ were hybridized in the same slide. In the $s c^{4}$ section, note the greatly reduced expression and the diminished clustering of the label. $(B)$ Section parallel to the main plane of a WD showing multiple clusters of $s c$-expressing cells on the presumptive notum (n) and dorsal wing base. Arrows indicate two sites of label seen only in the larger, and, presumably, older discs; they were not present in the sections used to construct the model of Fig. 3C. Arrowhead indicates a spot of label in the notopleural region, which is decreased strongly or absent in the $s c^{6}$ mutant discs. (D) Nearly frontal view of a computer-reconstructed WD, with two sections removed to show the highly complicated pattern of folds. The thin peripodial membrane, which would cover the front of the disc and whose developmental fate is unknown, largely has been removed to show the underlying, multiply folded epithelium. Note the three transverse folds $(1-3)$ and the complex fold separating the presumptive ventral wing surface from the pleura (arrowhead). Developing trachea, just below fold 1 and on the back of the model, is seen clearly. $(E)$ Selected section from the WD displayed in Fig. 3C showing hybridization in the presumptive regions of the ventral wing base $(\mathrm{v})$, wing margin $(\mathrm{w})$, dorsal radius $(\mathrm{dr})$, and trachea $(t r)$. This section is in approximately the same position as the highlighted section in $D$. Transverse folds are indicated by arrowheads. The complex fold $(c)$ appears simpler than in $D$, because subfolds are closed. $(\mathrm{tg})$ Tegula. All sections are reproduced to the same scale. Largest vertical dimension of disc in A is $0.35 \mathrm{~mm}$. Discs in $A-D$ and $F$ are oriented with the anterior margin to the left. 

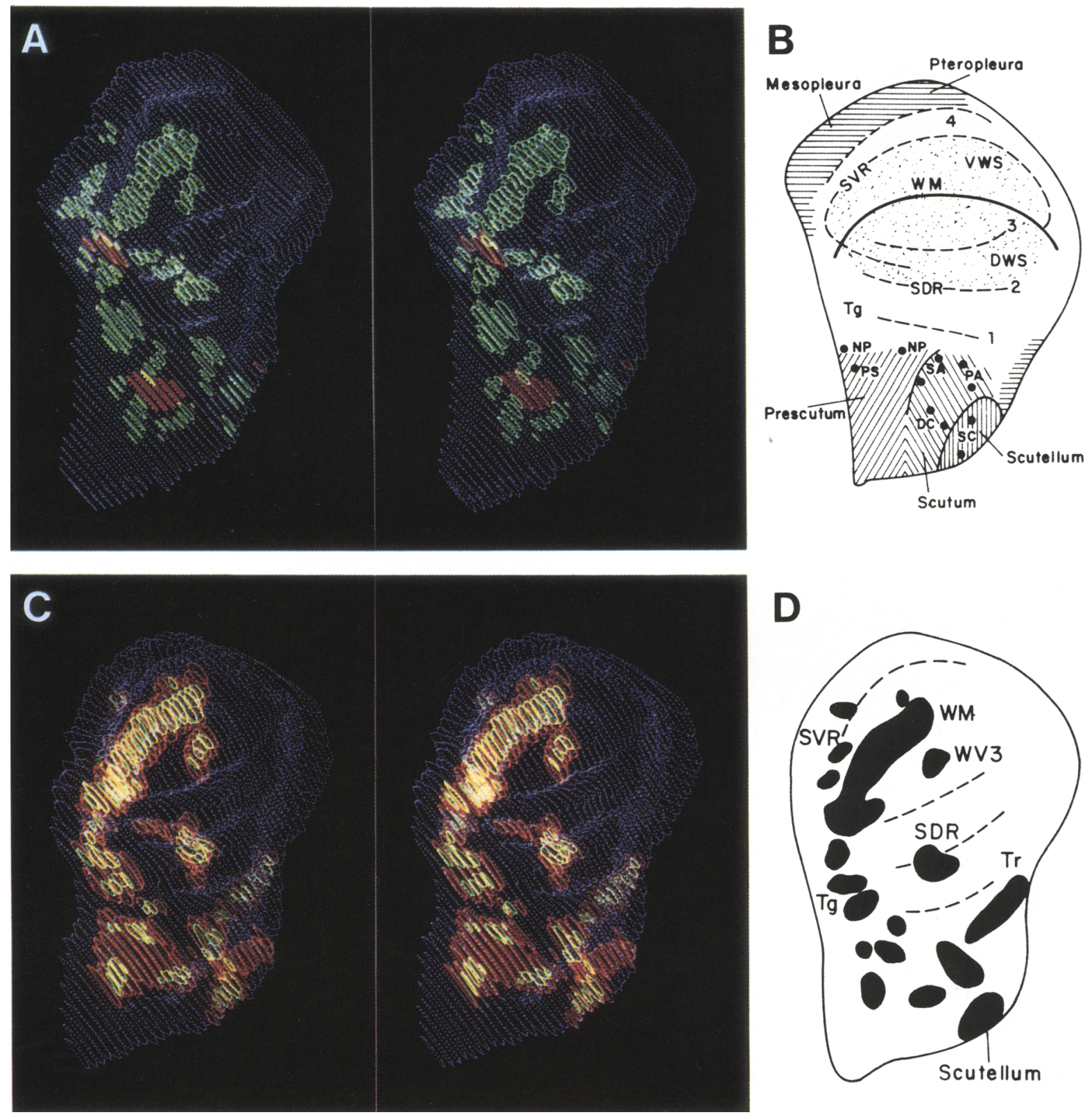

Figure 3. Stereo pairs showing WDs reconstructed from serial sections hybridized with $a c|A\rangle$ or $s c|C\rangle$ probes, fate map $\langle B\rangle$ of the WD, and schematic representation of $s c$ hybridized disc. Discs are shown with a slight tilt around the vertical axis. In the $s c$ probe hybridized disc $(C)$, yellow contours surround areas in sections where hybridization is maximal, whereas red contours delimit areas of low hybridization. The lower amount of hybridization obtained with the $a c$ probe $(A)$ made this distinction difficult to assess, so it has only been indicated in the few areas with red outlines. (B) A simplified fate map of the WD, taken from Bryant (1975), with indication of the localization of several presumptive regions for adult structures. (WM) Wing margin; (VWS and DWS) ventral and dorsal wing surface, respectively; (SVR and SDR) sensilla of the ventral and dorsal radius, respectively; (Tg) tegula. ( $\bullet$ ) Approximate positions of macrochaetae precursor cells. Standard chaetae nomenclature (Lindsley and Grell 1968; García-Bellido 1979) has been used: (DC) dorsocentrals; (NP) notopleurals; (PA) postalars; (PS) presutural; (SA) supraalars; (SC) scutellars. The presumptive notum reaches approximately up to fold 1 , and the presumptive dorsal hinge and base of the wing comprise approximately the area between folds 1 and 2 . Fold 3 is within the presumptive dorsal wing surface. An approximately semicircular fold (4), with a complicated array of subfolds (Fig. 2D), contains the presumptive ventral hinge region and separates the ventral wing surface from the mesothoracic pleura. $(D)$ Schematic representation of the $s c$ hybridized disc in a slightly different orientation than in $C$ to better illustrate all sites of label. (WV3) Wing vein 3 ; ( Tr) trachea. Note that expression of $a c$ and $s c$ occurs in the same areas of the disc, except the trachea which is labeled only by the $s c$ probe. 
wing radius and bears $>40$ sensilla campaniformia. The pattern on the presumptive notum is very complex (Figs. $2 \mathrm{~B}$ and $3 \mathrm{~A}, \mathrm{Cl}$ and given the number of clusters and the uncertainties in the fate map, it is difficult to assign labeled regions to individual organ(s). Still, the distribution of label is strongly reminiscent of the fate map. Part of the areas corresponding to the prescutum and scutum, which bear $\sim 130$ microchaetae in the adult, have a diffuse label. The expression occurring deep in fold 4 probably corresponds, at least in part, to the presumptive ventral wing radius, which bears 12 sensilla campaniformia. Although not specified in the fate map, the labeled clusters just below and above the middle of the wing margin may correspond to the third wing vein and adjacent cross vein which, together, bear four dorsal and one ventral sensilla. In summary, $a c / s c$ are expressed in all regions of the WD where SO precursors are known to be located. $s c$, but not $a c$, is expressed in a developing trachea, a site not known to have SO precursors, although it may have other nervous system elements.

\section{Early and late expression}

The earliest time $s c$ and $a c$ expressions have been detected in discs is at $40-48 \mathrm{hr}$ before puparium formation (BPF) (Fig. 4A). This time coincides with the earliest known determination of SOs in the WD derivatives (García-Bellido and Santamaría 1978). However, discs are small at this time and we have been unable to identify with certainty which individual discs are observed. Expression seems to occur in groups of cells, but only one or very few clusters are present in each disc, even in a tentatively identified WD (Fig. 4B). In contrast, an older WD disc, from a larva collected $23 \mathrm{hr} B P F$, shows several clusters of cells, indicating that the pattern of expression increases in complexity with the age of the disc (Fig. 4C).

After puparium formation (APF) discs undergo eversion making it very difficult to follow the modifications of individual sites of label. However, in white prepupae, just $1 \mathrm{hr}$ APF, the topology of the WD is still easily recognizable. Eversion of the wing has already begun. The band of label on the presumptive wing margin (Fig. 2A), which resolves into two parallel bands in slightly older discs (Fig. 5A), disappears in white prepupae (Fig. 5C). In contrast, label is still present in many other sites. At later times (18 hr APF), expression in many regions of disc derivatives is still recognizable and occurs in clusters of cells.

\section{ac/sc expression coincides with the early differentiation of SOs}

To verify the correlation between regions of $a c / s c$ expression and sites where SO precursors should be located, according to the WD fate map, we used transformant A37, which expresses $\beta$-galactosidase in peripheral nervous system (PNS) precursor cells (Ghysen and O'Kane 1989). In LTI-WDs, these precursors are beginning to express the enzyme (A. Ghysen, pers. comm.); consequently, many, but not all, SO precursors can be detected (Figs. 5B and 6B,C). Their location coincides with the regions of $a c / s c$ expression. Foremost examples are the double row of stained (Fig. 5B) and labeled (Fig. $5 \mathrm{~A}$ ) cells of the wing margin and the large cluster of $\mathrm{SO}$ precursors of the dorsal radius region. Stained cells in the presumptive notum probably correspond to macrochaetae precursors and lie within clusters of $a c / s c$ label (cf. Figs. $6 \mathrm{~B}, \mathrm{C}$ with $2 \mathrm{~B}$ and 3 ). We conclude that in LTIWDs, $a c$ and $s c$ are expressed when and in the same regions where part of the SO precursors are differentiating.

Next, we investigated whether $a c / s c$ products were necessary for SO precursors to reach the stage when $\beta$ galactosidase is synthesized. We stained WDs from larvae deficient for either $a c$ or $s c$. Control AS- $\mathrm{C}^{+}$larvae with only one copy of the A37 lacZ insertion (like the $a c$ - or $s c$-deficient larvae) showed weak staining. Still, the most visible precursors were those of the dorsal wing radius (Fig. 6E). These cells give rise to $>40$ sensilla campaniformia, most of which depend on $s c$, but not on $a c$, activity for development (Fig. 6A,D). Consistent with this dependence, the $a c$ deletion did not modify X-Gal staining detectably (not shown), but the $s c$ deletion suppressed it (Fig. 6F). Thus, many sensilla precursors of the dorsal radius require $s c$ activity for reaching the stage of differentiation defined by $l a c Z$ expression in the A37 transformant.

\section{Trans-regulation of ac/sc expression}

Genetic evidence suggests that the hairy $(h)$ and extramacrochaetae $(\mathrm{emc})$ loci negatively regulate $a c$ and $s c$ transcription (Moscoso del Prado and García-Bellido $1984 \mathrm{a}, \mathrm{b})$. However, in $h^{1}, e m c^{p e l}, e m c^{P 5 C}$ homozygous mutants, we have not detected modifications of the LTIWD $a c / s c$ patterns of expression (not shown). Moreover, the amounts of $a c$ and $s c$ RNA quantified in total RNA obtained from whole LTI larvae and 0- to 1-day-old pupae are not modified significantly in $h^{1} / h^{1}$ or $e m c^{1 /}$ TM2 individuals (not shown; TM2 carries a mutant allele for emc). Although these mutants harbor hypomorphic alleles, they show supernumerary SOs in ectopic positions. Null mutations are early lethals and, consequently, have not been analyzed.

In some morphogenetic genes, positive feedback regulation by their protein products has been invoked in maintaining and refining their patterns of expression (Hiromi and Gehring 1987; Bienz and Tremml 1988; Kuziora and McGinnis 1988). Because $a c$ and $s c$ encode related proteins, we examined the effect of the absence of both products on the transcription of $s c$. The double mutant $I n(1) s c^{10-1}$ does not synthesize $a c$ mRNA appreciably due to the In(1)ac ${ }^{3}$ breakpoint (Campuzano et al. 1985); also, it lacks complete sc protein due to an amber mutation in the $s c$-coding sequence (Villares and $\mathrm{Ca}$ brera 1987). Accordingly, $s c^{10-1}$ flies have extreme $a c$ and $s c$ phenotypes and are devoid of almost all SOs (GarcíaBellido 1979). In $s c^{10-1}$ LTI-WD, $s c$ RNA distribution was similar to that of the wild-type Oregon $\mathrm{R}$ strain, although it appeared to be several times more abundant, as judged from the number of silver grains on the ex- 


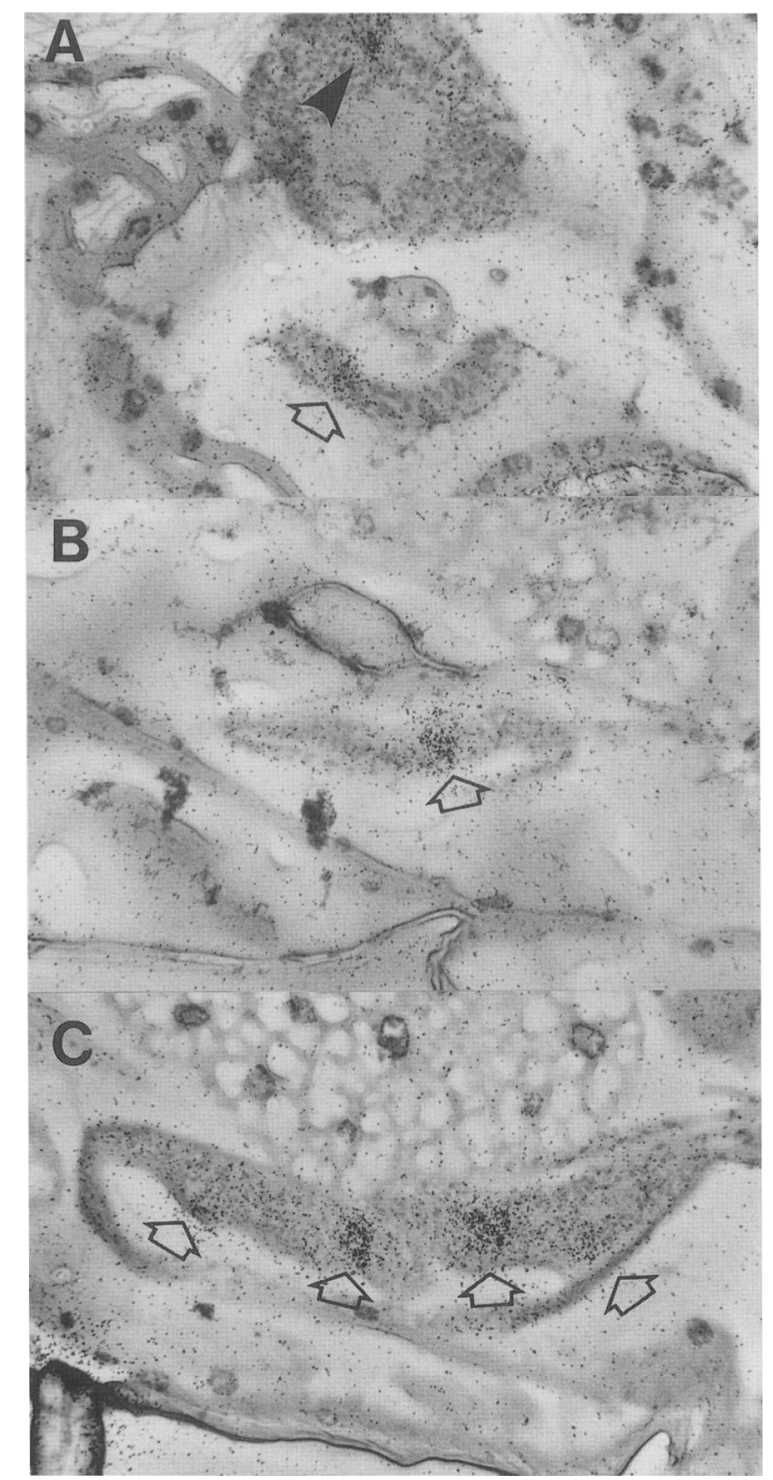

Figure 4. Expression of $s c$ in imaginal discs from third-instar larvae, $40(A), 32(B)$, and $24(C) \mathrm{hr}$ BPF. Imaginal disc shown in $A$ is probably an eye-antenna disc, whereas those shown in $B$ and $C$ are most likely WDs. These tentative identifications were based on positions of the discs within the larvae and their morphology. Arrows show sites of labeling on discs. Arrowhead shows the label on the optic lobe of the brain. Magnification, $1.35 \times$ with respect to Fig. 2 .

pressing sites (not shown). This result opens the possibility that the $s c$ product may, directly or indirectly, repress its own synthesis, although more trivial explanations, like differences in genetic background, are possible also. A repression of $s c$ by the ac product land vice versa) seems unlikely, because we did not observe modifications in the patterns of expression of either gene in the absence of the other (homozygous $D f(1) y^{3 P L} S c^{8 R}$ or $D f(1) s c^{8 L} S c^{4 R}$; not shown).

\section{Cis-regulation of sc expression}

Ruiz-Gómez and Modolell (1987) proposed that $s c$ is ac- tivated at specific sites of the imaginal discs by enhancer-like elements harbored in the $50 \mathrm{~kb}$ of AS-C DNA, downstream from the structural gene. To test this proposal, we examined the effect of removing these elements from the vicinity of the gene. We used the $\operatorname{In}(1) s c^{4}$ which has a breakpoint $7 \mathrm{~kb}$ downstream from the $s c$-coding region (Campuzano et al. 1985), and lacks most of the notum macrochaetae controlled by this gene (García-Bellido 1979). The sc RNA was much less abundant in $s c^{4}$ WDs than in the wild-type control, although some expression still occurred on the presumptive wing margin, wing vein 3 , dorsal radius, and tegula (Fig. $2 \mathrm{~A}, \mathrm{C})$. The pattern of the notum region was simplified, with several areas of diffuse label but without the welldefined, compact clusters of hybridizing cells characteristic of the wild-type pattern.

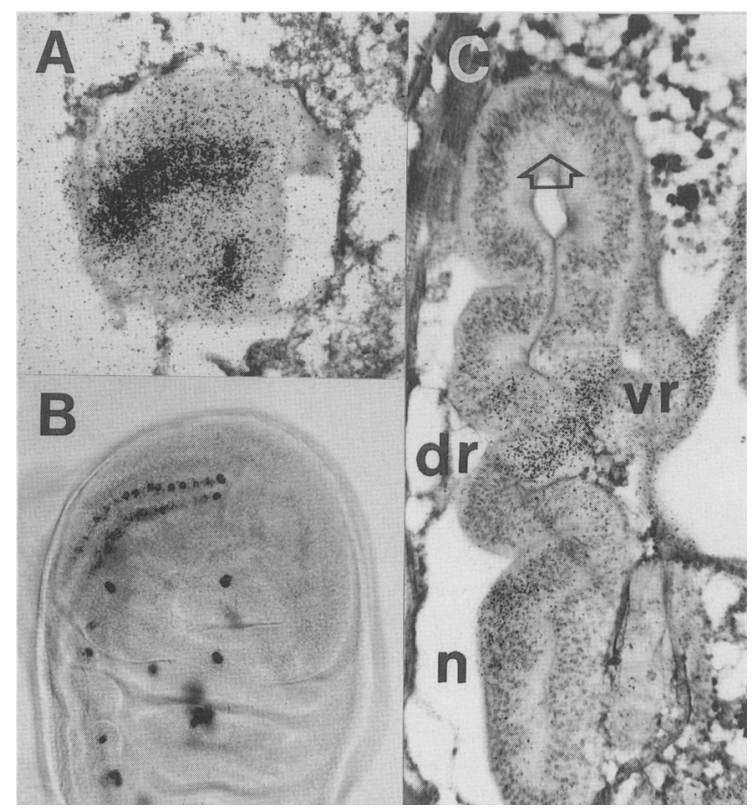

Figure 5. Expression of $s c(A)$ and location of SO precursors $(B)$ on the presumptive anterior wing margin of late third-instar WDs, and absence of $s c$ expression on the presumptive wing margin of a white prepupa WD $(C)$. $(A)$ Tangential section parallel to the main plane of the disc, showing the double row of label on the anterior wing margin. $(B)$ Expression of $\beta$-galactosidase in a disc of an A37 larva, showing a double row of SO precursors. In this disc, the wing margin is higher than that shown in Fig. $6 \mathrm{~B}$ and $\mathrm{C}$, indicating that the wing blade is beginning to evert and that the first disc is therefore developmentally older. The size of the disc in $A$, as estimated from other sections of the disc (not shown), indicates that the disc is approximately of the same developmental age as that shown in $B$. Note the topological correlation between the double rows of $s c$ expression and SO precursors in $A$ and $B .(C)$ Transverse section of a white prepupa disc. Arrow points to wing margin that lacks $s c$ RNA. Expression is detectable on the dorsal (dr) and ventral (vr) radius regions and on the presumptive notum (n). Note the eversion in progress of the wing blade (cf. with Fig. 2E). ac RNA, abundantly present in the LTI presumptive anterior wing margin, was also absent in this region of white prepupae discs (not shown). 
A more strict test was performed using the $s c^{6}$ mutation, a $16-\mathrm{kb}$ deletion located $25 \mathrm{~kb}$ downstream from the sc structural gene (Campuzano et al. 1985). Of the WD derivatives, only the anterior notopleural (ANP) macrochaeta is suppressed in this mutant (García-Bellido 1979). In the pattern of $s c$ expression, the only modification detected was the absence or strong reduction of a prominent spot located on the notopleural region of the disc (Fig.7; cf. with Fig. 2A,B). These data support the existence of elements controlling the spatial expression of $s c$ located far from this gene on the AS-C DNA.

\section{Discussion}

Of the four AS-C genes involved in neurogenesis, only $a c$ and $s c$ seem most relevant for the development of the adult pattern of SOs. Although this was inferred originally from genetic and molecular studies (García-Bellido 1979; Campuzano et al. 1985), it is shown here by $a c$ and $s c$ being expressed most abundantly in imaginal discs and by the spatial distributions of their transcripts which, at least for the LTI-WD, largely are restricted to the areas where SO precursors are found.

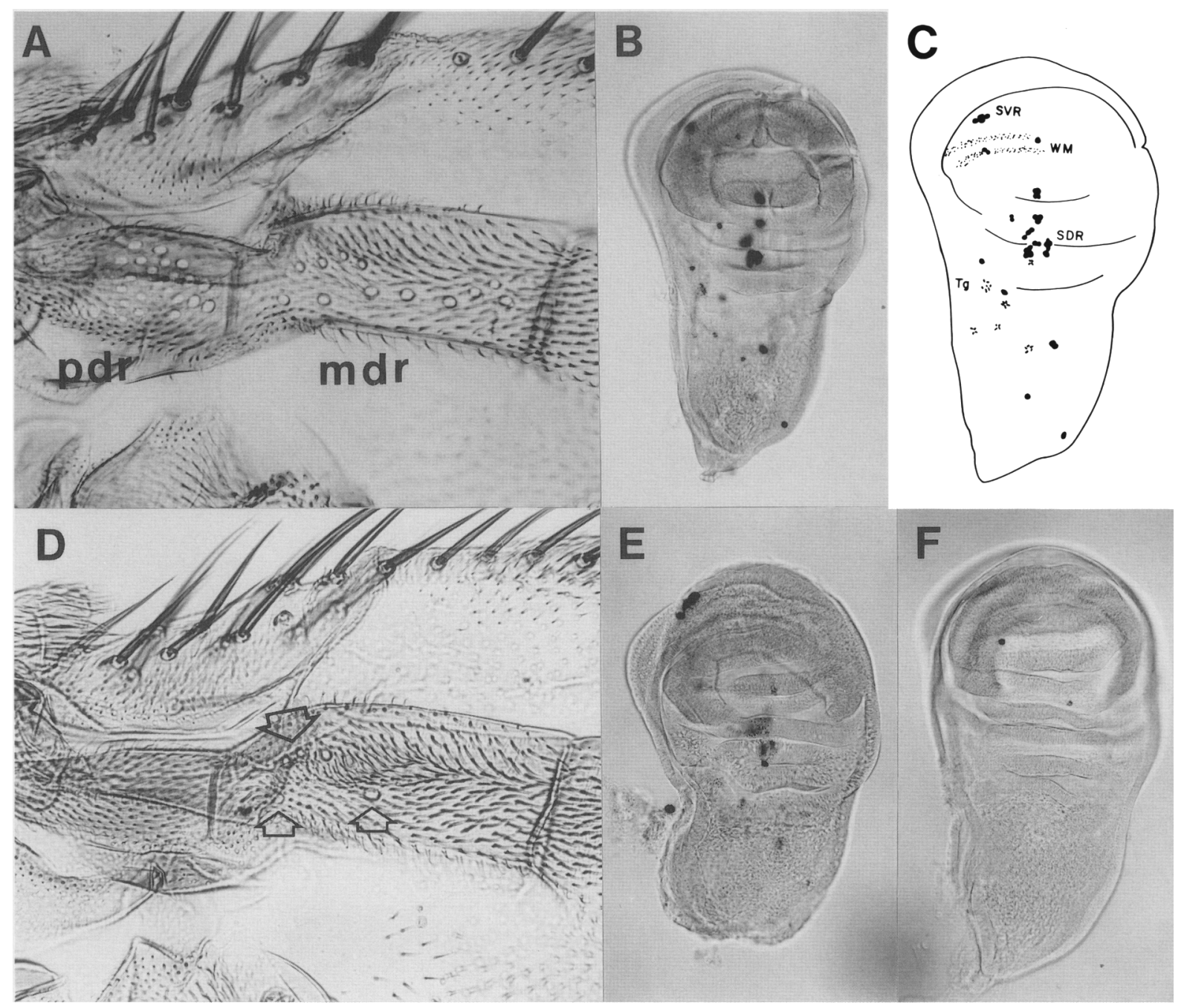

Figure 6. Sensilla on the dorsal radius of wild-type $(A)$ and $s c^{-}(D)$ flies and $\beta$-galactosidase expression in SO precursors of WD from A37 transformant in combination with wild-type $(B, C), a c^{-1}+(E)$ and $s c^{-}(F)$ alleles of the AS-C. $(A)$ In the wild-type, $\sim 40$ sensilla campaniformia (small circles or elipses) are distributed into several clusters on the proximal (pdr) and medial (mdr) dorsal radius. (D) Note that most of these sensilla, excluding a cluster of four, and two of a cluster of eight on the medial dorsal radius (arrowed), are absent in the $s c^{-}$deficiency $\left(D f(1) s c^{8 L} s c^{4 R}\right)$. In contrast, the $a c^{-}$deficiency $\left(D f(1) y^{3 P L} S c^{8 R}\right)$ only removes the cluster of four sensilla of the medial radius (not shown). Description of the molecular position of the breakpoints used to construct the deficiencies is given in Campuzano et al. (1985). ( $B, C)$ X-Gal staining of A37 transformant WD carrying two doses of the lac $Z$ gene. (C) Camera lucida drawing of the disc shown in $B$. Filled circles or ellipses indicated well-stained cells; dashes indicate cells or regions with incipient staining. Eighteen stained cells were present, at different levels, in the dorsal radius region (SDR). Other nomenclature is as described in the legend to Fig. 3B. $(E, F)$ X-Gal staining of heterozygous A37 transformant in combination with $D f(1) y^{3 P L} S c^{8 R /}+(E)$ and $D f(1) s c^{8 L} S c^{4 R}(F)$. Note that most of the staining disappears in the $s c^{-}$disc, indicating that $s c$ expression is essential for many cells to reach early stages of differentiation as PNS elements. 


\section{Significance of ac/sc expression for SO development}

It is known that $a c$ and $s c$ genes can promote neural commitment: Their ectopic expression switches cells from the epidermal to the SO developmental pathway ( $H w$ effect) (García-Alonso and García-Bellido 1986; Balcells et al. 1988). It is also known that lineage separation of SOs and epidermal precursor cells occurs in the WD, for the notum chaetae, at $\sim 40 \mathrm{hr}$ BPF /García-Bellido and Merriam 1971a). This time coincides with our earliest detection of $a c / s c$ transcripts in imaginal discs, thus suggesting that $a c$ and $s c$ products are involved in establishing this lineage separation. Also, it coincides approximately with the earliest time that mitotic recombination clones lacking $a c$ and $s c$ can be induced and still generate notum macrochaetae (García-Bellido and Santamaría 1978). Notum microchaetae appear in similar clones if induced $20 \mathrm{hr}$ BPF or later. However, the respective frequencies of macro- and microchaetae are several times lower than those of twin control clones (there is no preference for which chaetae can develop); frequencies increase at later times, but only become similar to those of the controls when clones are induced several hours APF. These results indicate that after cell lineage separation, there is a continuous requirement for $a c / s c$ products, over an interval $>40 \mathrm{hr}$, to maintain the neural state. This concept implies that the absence of $a c / s c$ products would either revert cells to an epidermal fate or cause cell death.

Note also that in the LTI stage, $a c / s c$ expression topologically coincides with SO precursors that are differentiating already, as judged by the expression of $l a c Z$ in the A37 transformant (Figs. 5B and 6C). These findings suggest that the late $a c / s c$ expression may also be important for SOs to accomplish the initial stages of differentiation. This role for the $a c / s c$ functions also has been suggested for the development of some leg sensory neurons (Jan et al. 1985) and the embryonic CNS. In this latter case, the deletion of the AS-C does not prevent many neuroblasts from appearing and dividing, but later, many progeny cells degenerate and die (Jiménez and Campos-Ortega 1979).

In summary, $a c / s c$ genes seem to induce and maintain neural state and promote initial stages of SO differentiation. These processes probably form a continous chain of events, involving activation/repression of sets of genes, which can be triggered at different times in different regions of the imaginal discs or even in different SO precursors of the same region. Irreversible neural state is probably attained during or after the initial stages of SO differentiation. A temporal separation between lineage segregation and irreversible neural state has been observed for embryonic neuroblasts (Technau et al. 1988). In agreement with $\mathrm{A}$. Ghysen, we propose the generic name 'proneural' for genes, such as $a c$, sc, and, probably, l'sc, ase, and da (Caudy et al. 1988a,b), whose activity commits cells to a neural developmental pathway. The products of these genes share sequence similarities (Villares and Cabrera 1987; Alonso and Cabrera 1988; Caudy et al. 1988b; F. González and J. Modolell, unpubl.). The name denotes the participation of the normal gene in generating nervous system elements, a basic difference from other genes whose mutant, loss-of-function condition causes a transformation toward neural elements (Artavanis-Tsakonas 1988; Campos-Ortega 1988).

\section{Control of ac and sc expression}

In a search for trans-regulatory genes of the AS-C, only two loci were found, $h$ and emc (Botas et al. 1982); these loci were proposed to act as negative regulators of the $a c$ and $s c$ functions, respectively (Botas et al. 1982; Moscoso del Prado and García-Bellido 1984a,b). Now we have found that $h$ and $e m c$ hypomorphic mutations do not affect either the patterns or the levels of transcription of $a c$ and $s c$ in LTI-WDs. These results are still compatible with $h$ and $e m c$ controlling $a c$ and $s c$ expression if, in hypomorphic mutations, the effects are too subtle for easy detection or regulation occurs at developmental stages other than the LTI larva. The results, nev-

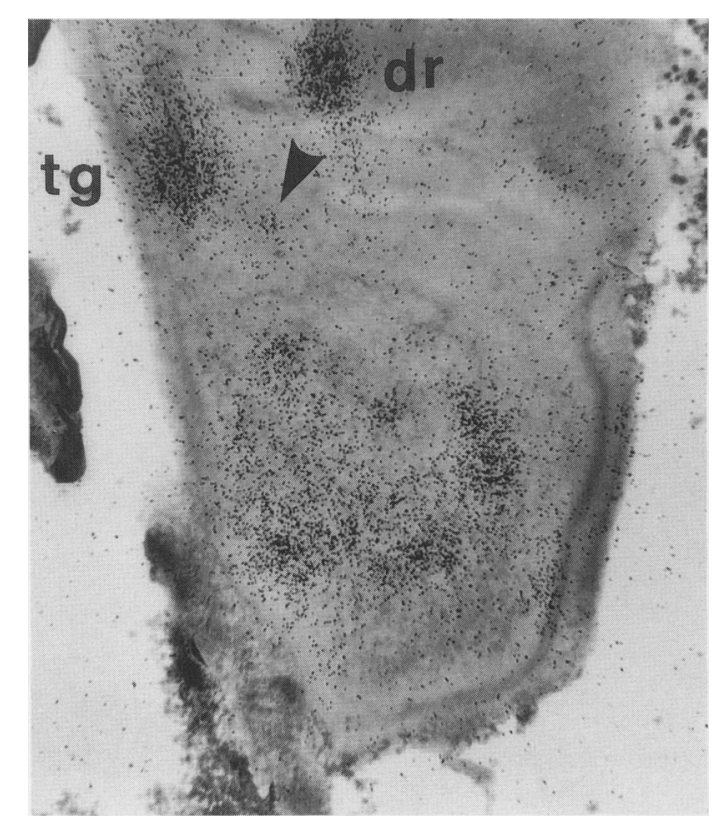

Figure 7. Expression of $s c$ in a late third-instar WD of the $s c^{6}$ mutant. Only the presumptive notum and dorsal wing-base regions of the disc are shown. Arrowhead indicates the notopleural area, where a most prominent spot of label is found in wild-type WDs (Fig. 2A,B), but it is very much reduced in this mutant. Of 11 WD examined, the spot was undetectable in five discs and was reduced very much in six discs. This was the only modification detected after examining serial sections of several discs. It is of interest that in $s c^{6}$ WDs carrying the $\mathrm{A} 37 \mathrm{lacZ}$ marker, a strong reduction in the frequency of a SO precursor, apparently located in the position of the reduced spot of $s c$ expression, has been observed (F. Huang, pers. comm.). Note, however, that the reduced spot and SO precursor seem to correspond to the posterior notopleural chaeta in the fate map (Fig. $3 \mathrm{~B}$ ), whereas the anterior notopleural chaeta is removed in the $s c^{6}$ mutation. Therefore, we suggest that an uncertainty in the fate map may be responsible for this apparent discrepancy. (tg) Tegula; $(\mathrm{dr})$ dorsal radius. Magnification, $1.62 \times$, with respect to Fig. 2. 
ertheless, are compatible also with a post-transcriptional interaction between the products of these loci and those of the AS-C. Regardless of the nature of the interaction, the reduction of $h^{+}$or $e m c^{+}$products in $h$ or emc mutants would make cells more sensitive to the neuralizing effects of the $a c / s c$ products.

Also, we have found that the absence of $a c$ or $s c$ does not modify the expression of the reciprocal gene, a result expected from the complementation of $a c$ and $s c$ mutations (García-Bellido 1979). Moreover, the presence of $a c$ and $s c$ products does not seem necessary for defining the areas where $s c$ is expressed. However, in the mutant tested $\left(\operatorname{In}(1) s c^{10-1}\right)$, a part of the $s c$ protein, containing a domain with homology to the myc proteins (Villares and Cabrera 1987), can still be synthesized. This truncated protein is inactive in SO development, but it is not known whether it has a role in generating the $s c$ pattern.

The deletion of a 16-kb segment of DNA, located 25 $\mathrm{kb}$ downstream of the $s c$ gene $\left(s c^{6}\right)$, causes a specific reduction of the expression of the gene in a single cluster of cells of the notopleural region of the WD, an alteration consistent with the phenotype of this mutation. This result supports the presence in the deleted region of an enhancer-like element(s) specifically activating the $s c$ gene in the notopleural cluster. Although we cannot rule out subtle effects at other sites of expression at the present level of resolution, apparently this element has one of the highest topological specificities described so far. As proposed elsewhere (Ruiz-Gómez and Modolell 1987; Ghysen and Dambly-Chaudière 1988|, a limited number of elements with different topological specificities would help additively in the construction of the spatial pattern of $s c$ expression. Consistent with this model, the separation of the $s c$ structural gene from its downstream DNA $\left(\operatorname{In}(1) s c^{4}\right)$, harboring several of these putative elements, causes a strong reduction in $s c$ mRNA abundance and a simplification of the pattern.

It is of interest that $a c$ and $s c$ genes are expressed in grossly the same areas of the WD and that the $s c$ region can be separated from the ac gene without modifying the $a c$ pattern $\left(\operatorname{In}(1) s c^{8 L} s c^{4 R}\right)$. Although we do not know whether the patterns of both genes also coincide at the single cell level, $a c$ and $s c$ regions may have functionally similar controlling elements. These elements may be interpreting a set of topological cues laid down by activators/repressors whose individual spatial distribution may not necessarily delimit the regions where the $a c / s c$ genes are active. Expression would occur only in cells with appropriate combinations of factors. This model, presupposes the existence of an evolving prepattern (Stern 1954; García-Alonso and García-Bellido 1988). Similar models have been proposed for the regulation of genes like $h$ and engrailed (DiNardo et al. 1988; Howard et al. 1988).

\section{Spatial restriction of ac/sc expression}

In LTI-WD, $a c / s c$ expression is restricted largely to regions where SO precursors are located. This restriction should be most important for imposing the topological constraints that define the regions where SOs can arise, for many WD epidermal cells are sensitive to the neuralizing effects of the $a c / s c$ products, even at this late stage. Thus, ectopic chaetae can be induced in $h^{-}$clones if the $h$ gene is removed up to $8 \mathrm{hr}$ BPF (García-Bellido and Merriam 1971b). Generalized expression of $s c$, induced in LTI larvae or early pupae, also causes differentiation of ectopic chaetae (M. Ruiz-Gómez and I. Rodríguez, unpubl.).

We have shown that $a c / s c$ expression occurs in groups of many cells that correspond well with regions where SOs develop. However, the correlation is not strict: For instance, expression in the scutellar area gives rise to two macrochaetae, whereas expression in a similar sized area like the dorsal radius gives rise to $>40$ sensilla campaniformia. Also, in $\mathrm{HW}$ mutants, generalized expression of $a c$ or $s c$ does not lead to generalized production of SOs, but to their presence in ectopic but allele-specific regions (García-Alonso and García-Bellido 1986; Balcells et al. 1988). Thus, the question arises as to whether the precise pattern of SOs derives from the less precise pattern of $a c / s c$ expression, and if so, how it is accomplished. Richelle and Ghysen (1979) have proposed a model in which a diffusible chaetogen $(a c / s c$ product) is synthesized by clusters of cells. Within each cluster, the cell that reaches a threshold of chaetogen concentration is committed to becoming a SO and inhibits neighboring cells from doing the same (Wigglesworth 1940|. Computer simulations showed that the compound response of a cluster of cells achieves a higher positional accuracy than that of the individual cells of the cluster. The observed distribution of $a c / s c$ RNAs in clusters fits with this model (see also Ghysen and Richelle 1979). However, the size and highly hydrophilic composition of the $a c / s c$ proteins make their diffusion very unlikely. Therefore, a more plausible possibility is that the distribution of $a c / s c$ products among the individual cells of a cluster might be controlled by active processes like cell interactions and that the resulting micropatterns would play a role in selecting the SO precursor cell(s). Notch, Delta, and other genes, which have loss-of-function neurogenic mutations and products structurally related to vertebrate epidermal growth factors, are candidates for mediating such interactions (Artavanis-Tsakonas 1988; Campos-Ortega 1988). Delta affects the embryonic expression of AS-C genes in the CNS (Brand and Campos-Ortega 1988). shaggy, whose mutations cause abundant appearance of extrachaetae (Simpson et al. 1988; Simpson and Carteret 1989), and emc (García-Alonso and García-Bellido 1988) are other candidates. It should be stressed that although $a c$ and $s c$ are expressed in similar areas, their micropatterns might be different. This difference could be related to the specific requirement of SOs in distinct positions for either product, because slight modifications of the pattern of expression of $s c$ may lead to replacement for the absence of ac (Balcells et al. 1988). Finally, another standing possibility is that $a c / s c$ expression only defines the general areas where SOs develop, and their precise number and position are determined by $a c / s c$-independent processes. 


\section{Experimental procedures}

\section{Drosophila stocks}

$e m c^{p e l}$ and $e m c^{P 5 c}$ are homozygous viable mutations at the $e x$ tramacrochaetae locus (Moscoso del Prado and García-Bellido $1984 \mathrm{a}, \mathrm{b}) . \mathrm{emc}^{\text {pel }}$ corresponds to a spontaneous mutation, and $e m c^{P 5 c}$ was found in an 'enhancer trap' search $\left\langle\mathrm{O}^{\prime} \mathrm{Kane}\right.$ and Gehring 1987). The latter contains a lacZ gene within a P transposable element inserted near the emc gene (J. Garrell, pers. comm.1. The A37 stock is a transformant line obtained by Cahir O'Kane, which expresses the lacZ gene in SO precursor cells (Ghysen and O'Kane 1989). $e m c^{P 5 c}$ and A37 stocks were kindly given to us by Stephen Kerridge and Alain Ghysen, respectively. Other stocks used are from the collection of A. GarcíaBellido and are described in Lindsley and Grell (1968).

\section{In situ hybridizations to larvae, pupae, and WD sections}

Larvae or pupae of the indicated developmental age were collected, fixed in Carnoy's fixative for $40 \mathrm{~min}$ after removing their posterior tips, dehydrated at room temperature, and embedded in paraffin wax (Ingham 1985). Sections (6 $\mu \mathrm{m}$ ) were cut and pretreated for hybridization as described (Ingham et al. 1985). $s c$ and $a c$ single-stranded RNA probes (prepared with ${ }^{35}$ Slabeled thioUTP, $1200 \mathrm{Ci} / \mathrm{mmole}$ / were synthesized on cDNA templates and hybridized to sections as described (Ingham et al. 1985). Exposures lasted 5-10 days. Hybridizations to sections from isolated WDs were performed as above, except that WDs were hand-dissected from LTI larvae in PBS and were fixed in $4 \%$ paraformaldehyde in PBS (20 min at room temperature) and $4 \%$ paraformaldehyde in $0.1 \%$ Triton X-100, $0.1 \%$ deoxycholate (20 min at room temperature). After washing with PBS, they were dehydrated and embedded in paraffin wax. Although there are similarities in the sequences of the $a c$ and $s c$ genes (Villares and Cabrera 1987), there was no cross-hybridization with the reciprocal probes under the conditions used.

\section{Histochemical staining for $\beta$-galactosidase activity}

Imaginal WDs were hand-dissected in PBS and fixed with $1 \%$ glutaraldehyde in PBS $\left(2 \mathrm{~min}\right.$ at $\left.0^{\circ} \mathrm{C}\right)$. Discs were washed twice in PBS and stained for $\beta$-galactosidase activity by incubation at $37^{\circ} \mathrm{C}$ for $20 \mathrm{hr}$ in $0.2 \%$ 5-bromo-4-chloro-3-indolyl- $\beta$-D-galactopyranoside (X-Gal), $5 \mathrm{mM} \mathrm{K}_{4}\left[\mathrm{Fe}(\mathrm{CN})_{6}\right], 5 \mathrm{mM} \mathrm{K}_{3}\left[\mathrm{Fe}(\mathrm{CN})_{6}\right]$ in PBS. After washing in PBS, discs were dehydrated, cleared in xylene, and mounted in Permount.

\section{Quantitation of transcripts}

Quantitation of $a c$ and $s c$ mRNA in whole RNA from larvae and pupae was carried out by an RNase-protection method, as described previously (Campuzano et al. 1986).

\section{Computer-aided three-dimensional reconstruction}

The computer-aided reconstructions of WDs were carried out as follows. Negatives of every section of each series were first rephotographed onto a $35-\mathrm{mm}$ film strip in alignment with one another, using an Image Combiner (Macagno et al. 1979). The film strip then was displayed on a screen, one frame at a time, and the boundaries of interest were drawn as close contours consisting of a series of connected vectors, using the CARTOS system (Macagno et al. 1979). Each feature, such as the boundary of the disc section or the boundaries of the regions of hybridization with a particular probe, was stored in a different file so that it could be displayed independently. The displays were generated on an Evans \& Sutherland PS-300 Graphics computer in color and photographed with Kodak Ektachrome film.

\section{Acknowledgments}

We are most grateful to A. García-Bellido, A. Ghysen, M. Levine, F. Jiménez, M. Ruiz-Gómez and colleagues in our laboratory for constructive criticisms on the manuscript; to $\mathrm{P}$. Simpson, J. Campos-Ortega, A. Ghysen, and Y.N. Jan for communication of unpublished results; to R. Griffin-Shea for introducing us to the subtleties of imaginal disc morphology; to S. Kerridge and A. Ghysen for stocks; to N. Necles for help in the generation of the computer reconstructions; to R. Sánchez for excellent photographic work; and to I. Rodríguez for technical assistance. S.R. is a fellow from the Instituto de Cooperación Iberoamericana (ICI). This work was supported by grants from Comisión Interministerial de Ciencia y Tecnología (CICYT), Consejo Superior de Investigaciones Científicas (CSIC); Caja de Ahorros y Monte de Piedad de Madrid to J.M., and the National Institutes of Health (NIH) grant HD-20954 to E.R.M. The institutional grants from Fondo de Investigaciones Sanitarias and Fundación Areces to the Centro de Biología Molecular are acknowledged. The reconstructions were carried out at the Columbia University computer graphics facility, which is supported by NIH grant RR0042.

\section{References}

Alonso, M.C. and C.V. Cabrera. 1988. The achaete-scute gene complex of Drosophila melanogaster comprises four homologous genes. EMBO I. 7: 2585-2691.

Artavanis-Tsakonas, S. 1988. The molecular biology of the Notch locus and the fine tuning of differentiation in Drosophila. Trends Genet. 4: 95-100.

Balcells, Ll., J. Modolell, and M. Ruiz-Gómez. 1988. A unitary basis for different Hairy-wing mutations of Drosophila melanogaster. EMBO /. 7: 3899-3906.

Bate, C.M. 1978. Development of sensory systems in arthropods. In Handbook of sensory physiology, (ed. M. Jacobson) vol. 9, pp.1-53. Springer Verlag, Berlin, New York.

Bienz, M. and G. Tremml. 1988. Domain of Ultrabithorax expression in Drosophila visceral mesoderm from autoregulation and exclusion. Nature 333: 576-578.

Botas, J., J. Moscoso del Prado, and A. García-Bellido. 1982. Gene-dose titration analysis in the search of trans-regulatory genes in Drosophila. EMBO J. 1: 307-310.

Brand, M. and J.A. Campos-Ortega. 1988. Two groups of interrelated genes regulate early neurogenesis in Drosophila melanogaster. Wilhelm Roux's Arch. Dev. Biol. 197: 457-470.

Bryant, P.J. 1975. Pattern formation in the imaginal wing disc of Drosophila melanogaster: Fate map, regeneration and duplication. I. Exp. Zool. 193: 49-78.

Campos-Ortega, J.A. 1988. Cellular interactions during early neurogenesis of Drosophila melanogaster. Trends Neurosci. 11: 400-405.

Campuzano, S., Ll. Balcells, R. Villares, L. Carramolino, L. García-Alonso, and J. Modolell. 1986. Excess function Hairy-wing mutations caused by gypsy and copia insertions within structural genes of the achaete-scute locus of Drosophila. Cell 44: 303-312.

Campuzano, S., L. Carramolino, C.V. Cabrera, M. Ruiz-Gómez, R. Villares, A. Boronat, and J. Modolell. 1985. Molecular genetics of the achaete-scute gene complex of $D$. melanogaster. Cell 40: 327-338. 
Caudy, M., E.H. Grell, C. Dambly-Chaudière, A. Ghysen, L.Y. Jan, and Y.N. Jan. 1988a. The maternal sex determination gene daughterless has zygotic activity necessary for the formation of peripheral neurons in Drosophila. Genes Dev. 2: $843-852$.

Caudy, M., H. Vaessin, M. Brand, R. Tuma, L.Y. Jan, and Y.N. Jan. 1988b. daughterless, a gene essential for both neurogenesis and sex determination in Drosophila, has sequence similarities to myc and the achaete-scute complex. Cell 55: 1061-1067.

Davis, R.L., H. Weintraub, and A.B. Lassar. 1987. Expression of a single transfected cDNA converts fibroblasts to myoblasts. Cell 51: 987-1000.

DiNardo, S., E. Sher, J. Heemskerk-Jongens, J.A. Kassis, and P.H. O'Farrell. 1988. Two-tiered regulation of spatially patterned engrailed gene expression during Drosophila embryogenesis. Nature 332: 604-609.

García-Alonso, L. and A. García-Bellido. 1986. Genetic analysis of Hairy-wing mutations. Wilhelm Roux's Arch. Dev. Biol. 195: $259-264$.

-1988. extramacrochaetae, a trans-acting gene of the achaete-scute complex of Drosophila involved in cell communication. Wilhelm Roux's Arch. Dev. Biol. 197:328338.

Garcia-Bellido, A. 1979. Genetic analysis of the achaete-scute system of Drosophila melanogaster. Genetics 91: 491-520.

García-Bellido, A. and J.R. Merriam. 1971a. Parameters of the wing imaginal disc development of Drosophila melanogaster. Dev. Biol. 24: 61-87.

- 1971b. Genetic analysis of cell heredity in imaginal discs of Drosophila melanogaster. Proc. Natl. Acad. Sci. 68: $2222-2226$.

García-Bellido, A. and P. Santamaría. 1978. Developmental analysis of the achaete-scute system of Drosophila melanogaster. Genetics 88: 469-486.

Ghysen, A. and C. Dambly-Chaudière. 1988. From DNA to form: The achaete-scute complex. Genes Dev. 2: 495-501.

Ghysen, A. and C. O'Kane. 1989. Neural enhancer-like elements as specific cell markers in Drosophila. Development 105: $35-52$.

Ghysen, A. and J. Richelle. 1979. Determination of sensory bristles and pattern formation in Drosophila. II. The achaete-scute locus. Dev. Biol. 70: 438-452.

Hiromi, Y. and W.J. Gehring. 1987. Regulation and function of the Drosophila segmentation gene fushi tarazu. Cell 50: $963-974$

Howard, K., P. Ingham, and C. Rushlow. 1988. Region-specific alleles of the Drosophila segmentation gene hairy. Genes Dev. 2: $1037-1046$.

Ingham, P.W. 1985. Genetic control of the spatial pattern of selector gene expression in Drosophila. Cold Spring Harbor Symp. Quant. Biol. 50: 201-208.

Ingham, P.W., K.R. Howard, and D. Ish-Horowicz. 1985. Transcription pattern of the Drosophila segmentation gene hairy. Nature 318: 439-445.

Jan, Y.N., A. Ghysen, I. Christoph, S. Barbel, and L.Y. Jan. 1985. Formation of neuronal pathways in the imaginal discs of Drosophila melanogaster. J. Neurosci. 5: 2453-2464.

Jiménez, F. and J.A. Campos-Ortega. 1979. On a region of the Drosophila genome necessary for central nervous system development. Nature. 282: 310 -312.

Kuziora, M.A. and W. McGinnis. 1988. Autorregulation of a Drosophila homeotic selector gene. Cell 55: 477-485.

Lech, K., K. Anderson, and R. Brent. 1988. DNA-bound fos proteins activate transcription in yeast. Cell 52: 179-184.
Lindsley, D.L. and E.H. Grell. 1968. Genetic variations of Drosophila melanogaster. Carnegie Inst. Wash. Publ. No. 627.

Macagno, E.R., C. Levinthal, and I. Sobel. 1979. Three-dimensional computer reconstruction of neurons and neuronal assemblies. Annu. Rev. Biophys. Bioeng. 8: 323-351.

Moscoso del Prado, J. and A. García-Bellido. 1984a. Genetic regulation of the achaete-scute complex of Drosophila melanogaster. Wilhelm Roux's Arch. Dev. Biol. 193: 242-245.

$1984 \mathrm{~b}$. Cell interactions in the generation of chaete pattern in Drosophila. Wilhelm Roux's Arch. Dev. Biol. 193: $246-251$

Murre, C., P.S. McCaw, and D. Baltimore. 1989. A new DNA binding and dimerization motif in immunoglobulin enhancer binding, daughterless, MyoD, and myc proteins. Cell 56: $777-783$.

O'Kane, C. and W.J. Gehring. 1987. Detection in situ of genomic regulatory elements in Drosophila. Proc. Natl. Acad. Sci. 84: 9123-9127.

Richelle, I. and A Ghysen. 1979. Determination of sensory bristles and pattern formation in Drosophila. I. A model. Dev. Biol. 70: 418-437.

Ruiz-Gómez, M. and J. Modolell. 1987. Deletion analysis of the achaete-scute locus of D. melanogaster. Genes Dev 1: $1238-1246$.

Simpson, P. and C. Carteret. 1989. A study of shaggy reveals spatial domains of expression of achaete-scute alleles on the thorax of Drosophila. Development 106: 57-66.

Simpson, P., M. El Messal, J. Moscoso del Prado, and P. Ripoll. 1988. Stripes of positional homologies across the wing blade of Drosophila melanogaster. Development 103: 391-401.

Stern, C. 1954. Two or three bristles. Am. Sci. 42: 213-247.

Technau, G.M., T. Becker, and J.A. Campos-Ortega. 1988. Reversible commitment of neural and epidermal progenitor cells during embryogenesis of Drosophila melanogaster. Wilhelm Roux's Arch. Dev. Biol. 197: 413-418.

Villares, R. and C.V. Cabrera. 1987. The achaete-scute gene complex of $D$. melanogaster: Conserved domains in a subset of genes required for neurogenesis and their homology to myc. Cell 50: 415-424.

Wigglesworth, V.B. 1940. Local and general factors in the development of 'pattern' in Rhodnius prolixus. I. Exp. Biol. 17: $180-200$. 


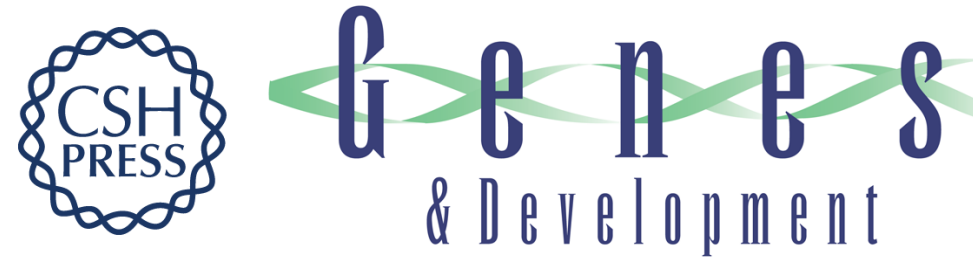

\section{Expression of achaete and scute genes in Drosophila imaginal discs and their function in sensory organ development.}

S Romani, S Campuzano, E R Macagno, et al.

Genes Dev. 1989, 3:

Access the most recent version at doi:10.1101/gad.3.7.997

References This article cites 44 articles, 14 of which can be accessed free at:

http://genesdev.cshlp.org/content/3/7/997.full.html\#ref-list-1

License

Email Alerting

Service

Receive free email alerts when new articles cite this article - sign up in the box at the top right corner of the article or click here.

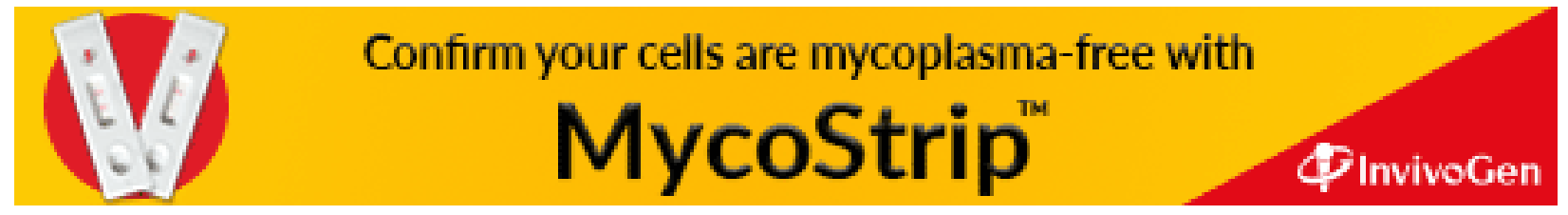

